\title{
Silicon photonic crystal cavities at near band-edge wavelengths
}

Cite as: Appl. Phys. Lett. 114, 091101 (2019); https://doi.org/10.1063/1.5067358

Submitted: 17 October 2018 . Accepted: 13 February 2019. Published Online: 04 March 2019

Salahuddin Nur (D), Hee-Jin Lim, Jeroen Elzerman, and John J. L. Morton
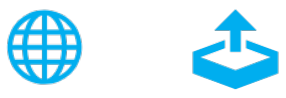

\section{ARTICLES YOU MAY BE INTERESTED IN}

The discrete noise of magnons

Applied Physics Letters 114, 090601 (2019); https://doi.org/10.1063/1.5088651

Photoresponsivity enhancement in monolayer $\mathrm{MoS}_{2}$ by rapid $\mathrm{O}_{2}$ :Ar plasma treatment Applied Physics Letters 114, 091103 (2019); https://doi.org/10.1063/1.5086726

A critical review of recent progress on negative capacitance field-effect transistors

Applied Physics Letters 114, 090401 (2019); https://doi.org/10.1063/1.5092684

\section{Applied Physics Reviews} Now accepting original research 


\title{
Silicon photonic crystal cavities at near band-edge wavelengths
}

\author{
Cite as: Appl. Phys. Lett. 114, 091101 (2019); doi: 10.1063/1.5067358 \\ Submitted: 17 October 2018 - Accepted: 13 February 2019. \\ Published Online: 4 March 2019
}

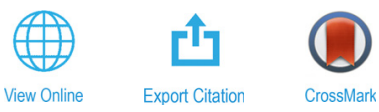

Salahuddin Nur, ${ }^{1,2, a)}$ (D) Hee-Jin Lim, ${ }^{1, b)}$ Jeroen Elzerman, ${ }^{1,2}$ and John J. L. Morton ${ }^{1,2}$

\begin{abstract}
AFFILIATIONS
'London Centre for Nanotechnology, University College London, London WC1H OAH, United Kingdom

${ }^{2}$ Department of Electronic and Electrical Engineering, University College London, London WC1E 7JE, United Kingdom

a) Author to whom correspondence should be addressed: salahuddin.nur.13@ucl.ac.uk. Present address: Institute of Electronics, Atomic Energy Research Establishment, Savar, Dhaka 1349, Bangladesh.

b) Present address: Korea Research Institute of Standards and Science, Dajeon 34113, South Korea.
\end{abstract}

\begin{abstract}
We demonstrate photonic crystal L3 cavities with a resonant wavelength of around $1.078 \mu \mathrm{m}$ on an undoped silicon-on-insulator, designed to enhance spontaneous emission from phosphorus donor-bound excitons. We have optimised a fabrication recipe using readily available process materials such as polymethyl methacrylate as a soft electron-beam mask and a Chemical Vapour Deposition grown oxide layer as a hard mask. Our bilayer resist technique efficiently produces photonic crystal cavities with a quality factor $(Q)$ of $\sim 5000$ at a wavelength of $1.078 \mu \mathrm{m}$, measured using cavity reflection measurements at room temperature. We observe a decrease in $Q$ as the cavity resonance shifts to shorter wavelengths $(Q \leq 3000$ at wavelengths $<1.070 \mu \mathrm{m})$, which is mostly due to the intrinsic absorption of silicon.
\end{abstract}

Published under license by AIP Publishing. https://doi.org/10.1063/1.5067358

Defect spins in solid state materials are attractive candidates for scalable implementation and integration of quantum information processing (QIP), ${ }^{1,2}$ metrology, ${ }^{3,4}$ and communication systems. ${ }^{5,6}$ For example, coherent spins in diamond and their interactions with photons have been exploited for optically mediated entanglement of matter-based systems. ${ }^{7}$ Although nitrogen-vacancy (NV) centres in diamond possess many attractive features that have underpinned key quantum information/communication demonstrations, some of the optical properties are sub-optimal (broad phonon sideband and spectral broadening), while thin-film growth and fabrication processes still need to be perfect. For such reasons, other materials systems combining excellent optical and spin memory properties with mature fabrication techniques are being explored to develop effective spin-photon interfaces. ${ }^{2,8-10}$ Amongst these have been vacancies in silicon carbide (SiC) and defects in silicon (Si). Silicon and $\mathrm{SiC}$ host defects and impurities with long spin coherence time ${ }^{9,11}$ and narrow linewidth emission of photons ${ }^{9,12,13}$ and permit coherent optical control of spins. ${ }^{14}$ These features, combined with the mature industrial techniques in manufacturing and on-chip integration, make such spins attractive for efficient multi-qubit coupling and realising large scale QIP systems. However, strong non-radiative processes in silicon-based host materials restrict fluorescence efficiency ${ }^{15,16}$ and indistinguishable single photon generation, ${ }^{9,17}$ thus limiting the potential of optical interfaces with most defects in silicon. This issue can, in principle, be addressed by engineering the local photonic environment in the host material: for example, incorporating photonic structures such as circular Bragg resonators $(\mathrm{CBRs})^{18}$ or photonic crystal cavities $(\mathrm{PCCs})^{19,20}$ can enhance photon emission and collection efficiency by several orders of magnitude, potentially allowing it to compete with non-radiative processes such as Auger recombination.

Enhanced light-matter interaction in PCCs ${ }^{19}$ has been demonstrated for various quantum emitters including NV centers in diamond $^{20}$ and rare-earth-doped crystals ${ }^{10}$ and quantum dots in $\mathrm{GaAs},{ }^{21}$ with observed improvements in the radiative emission. ${ }^{10,22}$ Similar schemes with PCCs can be utilised to enhance defect related emission in $\mathrm{Si}^{23}$ and $\mathrm{SiC}^{24,25}$ systems. Several defects such as shallow donors in silicon ${ }^{12,26}$ and divacancies, ${ }^{9,27}$ transition metal, ${ }^{28}$ and Ky5 color centre ${ }^{25}$ defects in $\mathrm{SiC}^{27}$ manifest spin-coupled optical emission with wavelength near $1.078 \mu \mathrm{m}$ (i.e., near the silicon band-edge). PCCs with a high ratio of $\mathrm{Q}$-factor to mode volume $\left(\mathrm{Q} / V_{m}\right)$ could be used to develop efficient spin-photon interfaces to such defects; however, experimental studies in this wavelength range on such materials are limited. In $\mathrm{SiC}$, planar PCCs with wavelengths in the range of $1100-1300 \mathrm{~nm}$ have been fabricated ${ }^{24,25}$ with $Q / V_{m} \sim 900-1500$ $(n / \lambda)^{3}$, but the band-gap of $S i$ is substantially smaller and there are significant challenges related to absorption when the PCC resonance 
approaches the band-gap energy. In addition, photon absorption from the host material leads to uncertainties in the photonic modes and the photonic band-gap as compared to non-dispersive materials. ${ }^{29}$ Aside from the cavities themselves, detection of weak optical signals near the silicon band-edge is made challenging due to the low quantum efficiency of the conventional $\mathrm{Si}$ detectors or the high dark count in InGaAs detectors. ${ }^{30}$ However, recent developments in waveguideintegrated superconducting nanowire single-photon detectors can provide a detection efficiency around $90 \%$ over a very wide range-from ultraviolet to mid-infrared wavelengths. ${ }^{31}$ Indeed, integration of such detectors with PCCstructures ${ }^{32}$ could lead to optically addressable, monolithic spin-based quantum systems in silicon.

Here, we design, fabricate, and study suspended Si PCCs, made from silicon-on-insulator (SOI) substrates, with wavelengths in the range of $1065-1085 \mathrm{~nm}$ at room temperature. We incorporate finetuning and band-folding in our L3 cavities in order to achieve high quality factors and better extraction of light, which could be utilised to enhance shallow impurity spontaneous emissions such as donor bound exciton $\left({ }^{31} \mathrm{P} \mathrm{D}^{0} \mathrm{X} \rightarrow \mathrm{D}^{0}\right)$ transitions in silicon. We have optimised a fabrication recipe using relatively inexpensive process materials in order to realise Si PCCs using conventional etching/pattern transfer processes. Finally, we used cross-polarisation confocal microscopy $^{33,34}$ with a broadband source and a spectrograph with a lownoise $\mathrm{Si}$ detector array to measure PCC reflection spectra, observing PCC Q-factors in the range of 2600-6200, increasing with the cavity wavelength.

L3 cavities are implemented by removing a row of three air holes from the hexagonal photonic crystal $(\mathrm{PhC})$ lattice with lattice constant a. We systematically study near $\mathrm{Si}$ band-edge resonant modes by fabricating L3 PCCs with lattice constants ranging from $a=240$ to $300 \mathrm{~nm}$. The L3 fundamental mode $\left(L 3^{\circ}\right)^{37}$ can be further engineered to improve $Q$ and light outcoupling, while keeping the mode volume below $0.9(\lambda / n)^{3}$. Q can be increased by changing the position and/or size of one or more side-holes adjacent to the cavity. ${ }^{38}$ The position displacement of any side hole from its original location in the lattice is indicated by a shift, $\Delta s_{i}$, and any absolute change in the corresponding hole radius by, $\Delta r_{\text {side, } i}$. Figure 1 (a) shows the SEM image of an L3 cavity where the position and the size of a pair of holes (marked in red) on either side of the cavity have been adjusted $\left(\Delta s_{1}=0.16 a\right.$ and $\left.\Delta r_{\text {side, } 1}=+0.06 a\right)$. Such a change in the design can produce a simulated $Q_{\text {des }}$ as high as $\sim 45000$. $^{3}$
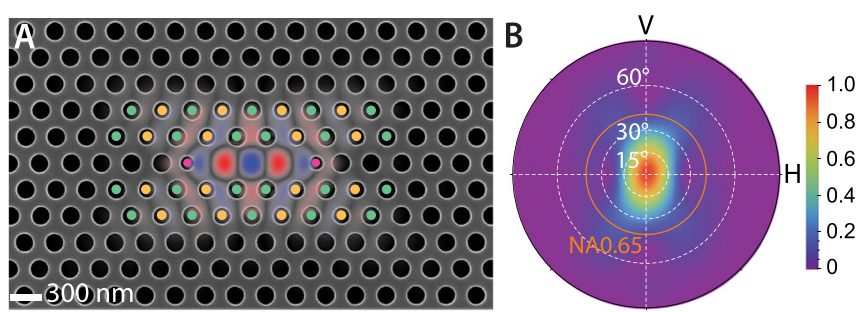

FIG. 1. (a) Scanning electron microscopy (SEM) image of a typical fabricated L3 cavity, with the superimposed simulated electric field profile based on the contour data extracted from the SEM image. Green and yellow circles indicate holes whose radii are adjusted to improve collection efficiency (see the main text). (b) Far field profile of the fundamental mode of the L3 cavity calculated by the contour FDTD method. $^{35}$
The vertical collection efficiency $(\eta)$ is improved by implementing a band folding scheme $\mathrm{e}^{39,40}$ in which gratings of periodicity $2 a$ are superimposed on the $\mathrm{PhC}$ lattice by modulating the radii of certain holes in the vicinity of the cavity. In Fig. 1(a), the radius of the green set of holes above and below the cavity is increased $(\Delta r=+0.02 a)$ from the regular air hole radius of $r_{0}=0.28 a$, while the radius of the yellow marked holes is reduced $(\Delta r=-0.02 a)$. The incorporation of such a hole-size modulation in the design limits the maximum achievable $Q_{\text {des }} \sim 1.5 \times 10^{4}$ but increases $\eta$ up to $\sim 0.8$ for an NA $=0.65$ [see Fig. 1(b)], where $\eta$ is estimated from the fabricated PCC structures using the contour finitedifference time-domain (FDTD) method ${ }^{35,36}$ and NA is the numerical aperture of the collection objective. These mode properties were simulated based on data extracted from SEM images of fabricated structures and are similar to those based on the idealised design. This indicates that fabrication errors are unlikely to be a significant contribution to collection losses. We have also implemented designs with three side hole shifts $\left(\Delta s_{1}=0.17 a, \Delta s_{2}=-0.025 a\right.$, and $\left.\Delta s_{3}=0.17 a\right)$ and a slightly modified modulation scheme in which the radii of the green holes remain at $r_{0}$, while the yellow have a larger radius $(\Delta r=+0.02 a)$. This modified hole modulation scheme, in principle, can further improve $Q$ while maintaining collection efficiencies of $\eta \sim 0.8$ for an NA $=0.65$.

We optimised fabrication process steps to transfer e-beam lithography $(\mathrm{EBL}$, at $30 \mathrm{kV})$ profiles into the thin $(220 \mathrm{~nm})$ Si device layer of the SOI chip with minimum distortions. Effective realisation of $\mathrm{Si}$ PhCs with small lattice constants $(<300 \mathrm{~nm})$ depends on the availability of a lithographic mask that can withstand plasma etching long enough to transfer the patterns efficiently to the $220 \mathrm{~nm} \mathrm{Si} \mathrm{layer.}{ }^{41} \mathrm{We}$ adopted a bilayer resist of polymethyl methacrylate (PMMA) $(250 \mathrm{~nm})$ and PECVD grown oxide layers $(300 \mathrm{~nm})$, which is less affected by proximity effects and can provide sufficient etch selectivity and anisotropy for the plasma etch steps under consideration. The process recipe includes two steps of reactive ion etching (RIE): $\mathrm{CHF}_{3} / \mathrm{Ar}$ plasma to transfer the pattern on the oxide layer and $\mathrm{CHF}_{3} / \mathrm{SF}_{6}$ plasma to etch the silicon layer using a PlasmaPro ${ }^{\circledR}$ NGP80 RIE tool from Oxford Instruments Plasma Technology. Conditions for anisotropic etching have been obtained by further adjusting RIE parameters including flow rates $\left(\mathrm{CHF}_{3}-25 \mathrm{sccm}\right.$ and $\left.\mathrm{Ar}-25 \mathrm{sccm}\right), \mathrm{RF}$ power $(150 \mathrm{~W})$, and pressure $(30 \mathrm{mT})$ for oxide etch and flow rates $\left(\mathrm{CHF}_{3}-58 \mathrm{sccm}\right.$ and $\left.\mathrm{SF}_{6}-25 \mathrm{sccm}\right)$, RF power $(150 \mathrm{~W})$, and pressure $(10 \mathrm{mT})$ for silicon etch. We run a cooling step ( $50 \mathrm{sccm}$ Ar flow without plasma) for 2 min after each $30 \mathrm{~s}$ long plasma etching step to avoid PMMA deformations by heat and repeat this cycle 24 times until the pattern penetrates through the $300 \mathrm{~nm}$ oxide hard mask layer. Finally, the pattern is transferred to the $220 \mathrm{~nm}$ Si device layer using plasma etch along with the oxide hard mask. To release the suspended membrane containing the $\mathrm{PhC}$, we undercut the buried oxide (BOX) layer of the SOI chip and remove remaining oxide masks together with hydrofluoric acid (HF). The major process steps in the optimised fabrication recipe are shown in Fig. 2. When the fabricated devices are inspected under SEM, it is found that fabrication errors are small. However, the hole radii in the silicon membrane were larger than the intended values by less than $10 \mathrm{~nm}$. This systematic error in the fabricated hole shapes was mitigated by reducing the hole radii in the EBL mask, which produced good PhCs with lattice constants $a$ between 240 and $300 \mathrm{~nm}$.

The fabricated Si PCCs were characterised by cavity reflection measurements using a cross-polarisation confocal setup shown in Fig. 3(a). An optical image of a PCC (a bright dot in the centre of the 


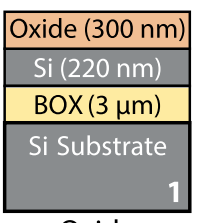

Oxide deposition

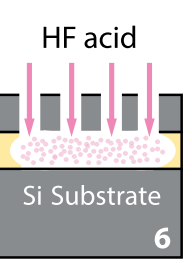

BOX removal

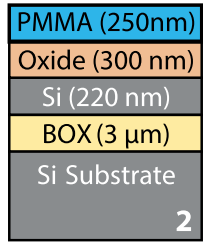

PMMA

coating

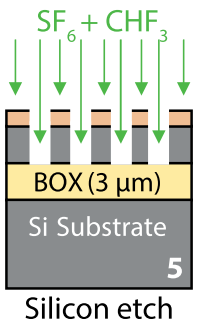

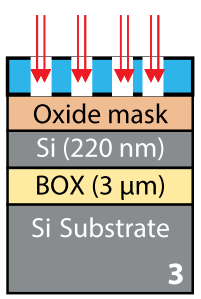

E-beam Lithography

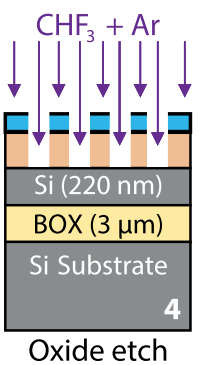

FIG. 2. Summary of the process steps for fabricating Si PCCs with the bilayer [PMMA/Oxide (PECVD)] resist (see the text for full details).

rectangle) captured using a CMOS camera (CM) is shown in Fig. 3(b). The cavity reflection signal was collected into a single mode polarisation maintaining fibre (PMF) in a confocal configuration where the point spread function is matched with the fibre mode, as shown in Fig. 3 (c), with a spatial resolution ${ }^{42}$ of $R_{\text {spatial }} \approx 0.6 \lambda_{\text {exc }} / \mathrm{NA} \approx 1 \mu$ m determined for a high numerical aperture $(\mathrm{NA}=0.65)$ and an excitation wavelength $\left(\lambda_{\text {exc }}\right)$ of $\sim 1.078 \mu \mathrm{m}$. The maximum coupling efficiency was measured to be $70 \%$ using the Gaussian beam collimated from a single mode fibre. ${ }^{43}$ The cross-polarisation setup was implemented by setting the two polarisers, POL1 and POL2 in orthogonal directions ${ }^{33,34}$ [Fig. 3(a)]. A suppression ratio around $10^{6}$ has been measured using this system, which in turn allows us to select reflections associated with the cavity mode. The bright spot shown in the camera image [Fig. 3(b)] also contains signals from higher order modes and tails of the cavity resonance, resolved with a signal-to-noise ratio (SNR) of $\sim 35$. In Fig. $3(\mathrm{c})$, the black trace is the point spread function/illumination profile from the source observed at the polarisation-maintaining fibre (PMF) end and the grey area denotes the collection by the PMF. The orange and red traces in Fig. 3(d) are the focussed field intensities of the $L 3^{\circ}$ mode along $x$ and $y$-axes, respectively. The $L 3^{\circ}$ field profile at the PMF end is calculated from field amplitudes and phases of the simulated farfield profile ${ }^{42}$ of the cavity [Fig. 1(b)]. Now, cavity scattering makes the focused spot spread wider along the $x$-axis (orange trace) than along the $y$-axis (red trace) at the end of the collection PMF. This gives a broader shape for the $L 3^{\circ}$ field profile along the $x$-axis [orange trace in Fig. 3(d)] than the collection by the PMF [grey shaded curves in Figs. 3(c) and 3(d)] and causes a mode mismatch with the PMF. From the traces in Fig. 3(d), we extract a mode mismatch of $\sim 30 \%$. Finally, by taking into account the collection efficiency of PCCs $(\eta \sim 80 \%)$, path losses in the optical setup, PMF coupling efficiency, etc., a total coupling efficiency of $\sim 9 \%$ has been estimated for our confocal setup.

An Acton SP-2750 Princeton Instruments spectrometer with a focal length of $0.75 \mathrm{~m}$ was used to capture the spectrum of the
A
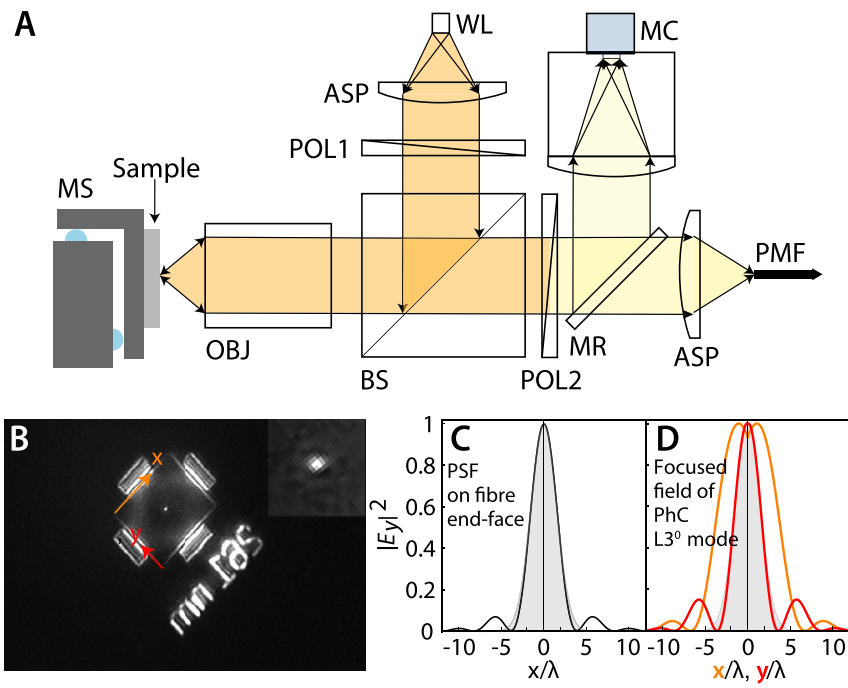

FIG. 3. (a) Confocal microscopy setup with cross-polarisation to measure the PCC reflection spectrum. (b) The inset shows an optical image of a PCC (appearing as a bright dot in the centre of the rectangle) captured by a high sensitivity, monochrome camera (MC). The MC was incorporated for focussing/sample surface inspection and placed after an analyser (POL2) which is aligned orthogonal to the polariser (POL1). Definitions used are MS: motorised stage, OBJ: objective lens, BS: nonpolarising beam splitter, ASP: aspheric lens, WL: white light source, MR: flippable mirror, and PMF: polarisation-maintaining fibre. (c) The point spread function (PSF) at the PMF end-face. (d) The focused field intensity based on the far-field of the cavity mode [see Fig. 1(b)]. The orange and red curves denote the focused field intensity distribution along $x$ and $y$-directions, respectively. The Grey shaded curves in (c) and (d) are mode profiles of the fibre mode.

collected signal. We used a $300 \mathrm{~g} / \mathrm{mm}$ blaze grating optimised for wavelengths around $1 \mu \mathrm{m}$, which can provide a resolution of approximately $150 \mathrm{pm}$ around the ${ }^{31} \mathrm{P} \mathrm{D}^{0} \mathrm{X} \rightarrow \mathrm{D}^{0}$ transition wavelengths. The dispersed light from the grating was detected by a Si-based CCD array (PyLoN System Silicon CCD Camera), enabling the measurement of quality factors up to $\sim 10000$. The output power of the LED source used (M1050D1, Thorlabs) peaks at $50 \mathrm{~mW}$ but varies considerably across the measurement window of interest (1060-1090 nm), falling to about $10 \mathrm{~mW}$ at the ${ }^{31} \mathrm{P} \mathrm{D}^{0} \mathrm{X} \rightarrow \mathrm{D}^{0}$ transition wavelength.

The results of cavity reflection measurements at room temperature are summarised in Fig. 4. For a single side-hole shifted L3 cavity with $a=262 \mathrm{~nm}$, we observe the fundamental resonant mode $\left(L 3^{\circ}\right)$ appearing at a wavelength of $\sim 1077 \mathrm{~nm}$, which closely matches the $\mathrm{D}^{0} \mathrm{X}$ transitions in silicon. The designed $Q$ for this $L 3^{\circ}$ mode is $Q_{\mathrm{des}} \approx 29000$, while the Lorentzian fit to the measured data gives an experimental $Q_{\mathrm{ex}} \approx 5000$. Such a mismatch is often attributed to fabrication or structural imperfections in the PCC; ${ }^{44}$ however, for near band-edge Si PCCs, the intrinsic material absorption can also be a dominant loss mechanism and this was not accounted for in the simulations of the idealised structures. Silicon has an absorption coefficient $\gtrsim 2 \mathrm{~cm}^{-1}$ near the bandedge, at room temperature, ${ }^{45,46}$ giving an upper bound of only a few thousand for achievable PCC $Q$ values. To investigate this in greater depth, we studied three side-hole shifted L3 cavities, designed for $Q_{\mathrm{des}}$ $\approx 50000$, across a lithographic tuning range with a step size of $3 \mathrm{~nm}$.

Near Si band-edge resonant modes for the three side-hole shifted L3 cavities are shown in Fig. 4(b), with selected fundamental 

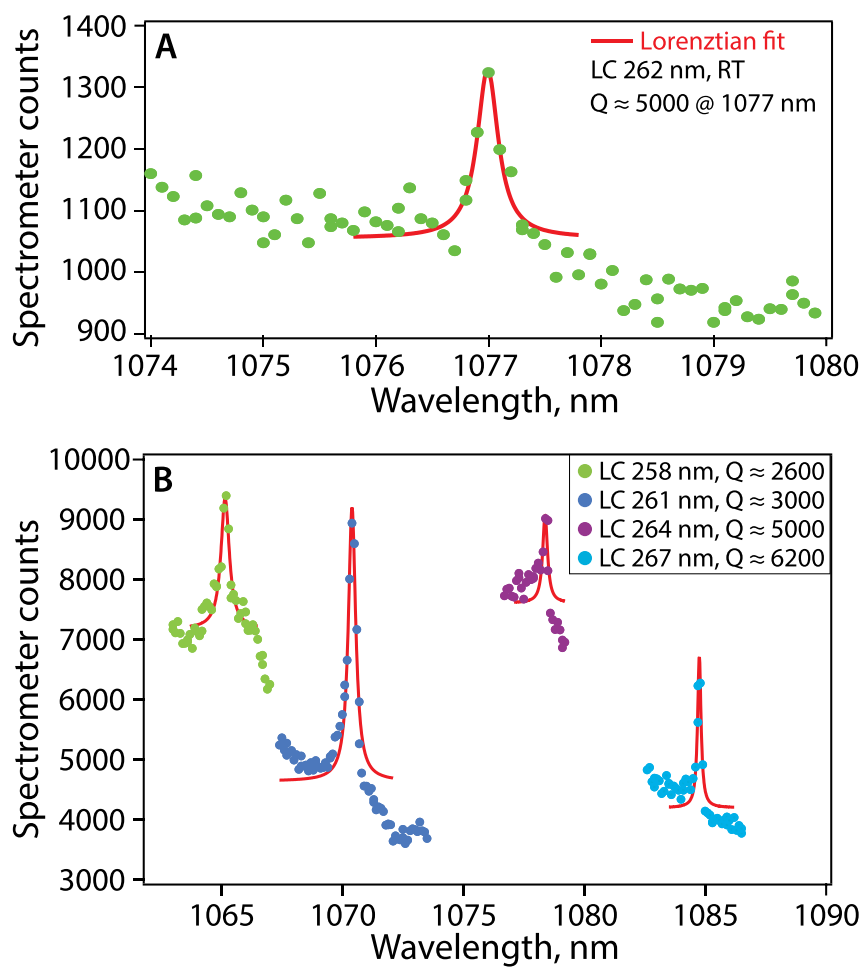

FIG. 4. Room temperature cavity reflection spectra measured using a crosspolarisation setup. Lorentzian fits to $L 3^{\circ}$ modes reveal quality factors for $L 3$ PCCs tuned with (a) single side-hole shift $\left(\Delta s_{1}=0.16 \mathrm{a}\right.$ and $\left.\Delta r_{\text {side1 }}=0.06 \mathrm{a}\right)$ and (b) three side-hole shifts $\left(\Delta s_{1}=0.17 a, \Delta s_{2}=-0.025 a\right.$ and $\left.\Delta s_{3}=0.17 a\right)$ for a collection efficiency of $\eta \sim 0.8$.

resonances $\left(L 3^{\circ}\right)$ at $1065 \mathrm{~nm}, 1070.4 \mathrm{~nm}, 1078.4 \mathrm{~nm}$, and $1084.8 \mathrm{~nm}$ for L3 cavities with lattice constants of $258 \mathrm{~nm}, 261 \mathrm{~nm}, 264 \mathrm{~nm}$, and $267 \mathrm{~nm}$, respectively. The quality factors $\left(Q_{\mathrm{ex}}\right)$ extracted from measurement data are $\sim 2600, \sim 3000, \sim 5000$, and $\sim 6200$, growing with the increasing $L 3^{\circ}$ wavelength. At room temperature, optical absorption in $\mathrm{Si}$ drops gradually with decreasing photon energies below the bandgap, ${ }^{45,46}$ consistent with our measurements. We also note that both Si PCC designs (single- and three-side-hold shifts) show $Q_{\mathrm{ex}} \approx 5000$ for $L 3^{\circ}$ near ${ }^{31} \mathrm{P} \mathrm{D}^{0} \mathrm{X}$ transition wavelengths $(\sim 1078 \mathrm{~nm})$, suggesting that losses in our near band-edge cavities at room temperature are dominated by intrinsic material absorption in $\mathrm{Si}$. Absorption at these wavelengths in Si drops significantly at cryogenic temperatures, ${ }^{45,46}$ and so, significantly higher Q values can be expected. Indeed, the relative effects of absorption and fabrication imperfections on suppressing $Q_{\mathrm{ex}}$ for wavelengths near the Si band-edge could be resolved in future studies comparing PCCs at cryogenic and room temperatures.

The total oscillator strength of ${ }^{31} \mathrm{P} \mathrm{D}^{0} \mathrm{X}$ transition in silicon is $7.1 \times 10^{-6}$, and the radiative lifetime is $\sim 2 \mathrm{~ms}{ }^{15,47}$ Low temperature $\mathrm{PL}$ measurements on the ${ }^{31} \mathrm{P}$ doped float zone $(\mathrm{Fz})$ type $\mathrm{Si}$ sample, using a similar measurement setup, have shown the detection of approximately 1000 effective ${ }^{31} \mathrm{P} \mathrm{D}^{0} \mathrm{X}$ emitters in $\mathrm{Si}$ (ensemble linewidth of $\sim 0.1 \mathrm{~nm}$ ), suggesting that an enhancement of around three orders of magnitude in the total emission efficiency would be sufficient to optically detect single ${ }^{31} \mathrm{P}$ donors in silicon. In principle, a Purcell enhancement $^{48}\left(F_{\mathrm{p}}\right)$ of $\sim 1000$ can be obtained from a cavity with $Q \sim 10^{4}$ and $V_{\text {mode }} \sim(\lambda / n)^{3}$. However, implementation of the band folding in our L3 PCC designs enhanced the collection ( $\eta_{\text {outcouple }}$ $\approx 80 \%$ ) by an order of magnitude which relaxes the requirement of a high Purcell enhancement, and the required three orders of magnitude enhancement in the total emission can be obtained from an $F_{\mathrm{P}}$ of $\sim 100$ with a collection efficiency of $\eta_{\text {outcouple }} \approx 80 \%$. Thus, the Qfactors of around 5000 that we measure at room temperature should be more than sufficient for detecting optical emission from spins down to the single or few donor/defect level in $\mathrm{Si}^{12,26}$ or $\mathrm{SiC}^{27,49}$

In summary, we have optimised a low cost fabrication process recipe for realising Si PCCs and efficiently fabricated L3 cavities with resonances near silicon band-edge wavelengths. Room temperature optical characterisation of fabricated cavities unveils an absorptionlimited $Q$ of 5000 with a collection efficiency close to $80 \%$ around $1078 \mathrm{~nm}$. Such near band-edge Si PCCs may play an important role in realising efficient spin-photon interfaces in $\mathrm{Si}$ and $\mathrm{SiC}$ systems.

We thank the London Centre for Nanotechnology cleanroom staff for their technical support. We thank G. Matmon, UCL, for the use of experimental equipment and insightful comments and T. F. Krauss and C. Reardon, University of York, for providing the SOI wafer and for useful discussions. The research leading to these results has received funding from the European Research Council under the European Union's Seventh Framework Programme (No. FP7/2007-2013)/ERC Grant Agreement No. 279781 (ASCENT) and Horizon 2020 research and innovation programme [Grant Agreement No. 771493 (LOQO-MOTIONS)]. We also acknowledge financial support from EPSRC and UCL Engineering.

\section{REFERENCES}

${ }^{7}$ T. D. Ladd, F. Jelezko, R. Laflamme, Y. Nakamura, C. Monroe, and J. L. O'Brien, Nature 464, 45 (2010).

${ }^{2}$ L. Gordon, J. R. Weber, J. B. Varley, A. Janotti, D. D. Awschalom, and C. G. Van de Walle, MRS Bull. 38, 802 (2013).

${ }^{3}$ C. Bonato, M. S. Blok, H. T. Dinani, D. W. Berry, M. L. Markham, D. J. Twitchen, and R. Hanson, Nat. Nanotechnol. 11, 247 (2016).

${ }^{4}$ S. A. Tarasenko, A. V. Poshakinskiy, D. Simin, V. A. Soltamov, E. N. Mokhov, P. G. Baranov, V. Dyakonov, and G. V. Astakhov, Phys. Status Solidi B 255, 1700258 (2018).

${ }^{5}$ M. S. Blok, N. Kalb, A. Reiserer, T. H. Taminiau, and R. Hanson, Faraday Discuss. 184, 173-182 (2015).

${ }^{6}$ S. Johnson, P. R. Dolan, and J. M. Smith, Prog. Quantum Electron. 55, 129 (2017).

${ }^{7}$ H. Bernien, B. Hensen, W. Pfaff, G. Koolstra, M. S. Blok, L. Robledo, T. H. Taminiau, M. Markham, D. J. Twitchen, L. Childress, and R. Hanson, Nature 497, 86 (2013).

${ }^{8}$ M. Atatüre, D. Englund, N. Vamivakas, S.-Y. Lee, and J. Wrachtrup, Nat. Rev. Mater. 3, 38-51 (2018).

${ }^{9}$ D. J. Christle, P. V. Klimov, C. F. de las Casas, K. Szász, V. Ivády, V. Jokubavicius, J. U. Hassan, M. Syväjärvi, W. F. Koehl, T. Ohshima, N. T. Son, E. Janzén, Á. Gali, and D. D. Awschalom, Phys. Rev. X 7, 021046 (2017).

${ }^{10}$ T. Zhong, J. M. Kindem, E. Miyazono, and A. Faraon, Nat. Commun. 6, 8206 (2015).

${ }^{11}$ M. Steger, K. Saeedi, M. L. W. Thewalt, S. Simmons, H. Riemann, N. V. Abrosimov, P. Becker, and H. J. Pohl, Science 336, 1280 (2012).

${ }^{12}$ A. Yang, M. Steger, D. Karaiskaj, M. L. W. Thewalt, M. Cardona, K. M. Itoh, H. Riemann, N. V. Abrosimov, M. F. Churbanov, A. V. Gusev, A. D. Bulanov, A. K. Kaliteevskii, O. N. Godisov, P. Becker, H. J. Pohl, J. W. Ager, and E. E. Haller, Phys. Rev. Lett. 97, 227401 (2006).

${ }^{13}$ M. Steger, T. Sekiguchi, A. Yang, K. Saeedi, M. E. Hayden, M. L. W. Thewalt, K. M. Itoh, H. Riemann, N. V. Abrosimov, P. Becker, and H. J. Pohl, J. Appl. Phys. 109, 102411 (2011). 
${ }^{14}$ O. V. Zwier, D. óShea, A. R. Onur, and C. H. van der Wal, Sci. Rep. 5, 10931 (2015).

${ }^{15}$ W. Schmid, Phys. Status Solidi B 84, 529 (1977).

${ }^{16}$ H. Sumikura, K. Nishiguchi, Y. Ono, A. Fujiwara, and M. Notomi, Opt. Express 19, 25255 (2011).

${ }^{17}$ M. Pelton, C. Santori, J. Vuckovic, B. Zhang, G. S. Solomon, J. Plant, and Y. Yamamoto, Phys. Rev. Lett. 89, 233602 (2002).

${ }^{18}$ M. Davanço, M. T. Rakher, D. Schuh, A. Badolato, and K. Srinivasan, Appl. Phys. Lett. 99, 041102 (2011).

${ }^{19}$ J. D. Joannopoulos, P. R. Villeneuve, and S. Fan, Nature 386, 143 (1997).

${ }^{20}$ A. Faraon, C. Santori, Z. Huang, V. M. Acosta, and R. G. Beausoleil, Phys. Rev. Lett. 109, 033604 (2012).

${ }^{21}$ A. Reinhard, T. Volz, M. Winger, A. Badolato, K. J. Hennessy, E. L. Hu, and A. Imamoglu, Nat. Photonics 6, 93 (2012).

${ }^{22}$ C.-M. Lee, H.-J. Lim, C. Schneider, S. Maier, S. Höfling, M. Kamp, and Y.-H. Lee, Sci. Rep. 5, 14309 (2015).

${ }^{23}$ K.-M. C. Fu, T. D. Ladd, C. Santori, and Y. Yamamoto, Phys. Rev. B 69, 125306 (2004).

${ }^{24}$ G. Calusine, A. Politi, and D. D. Awschalom, Appl. Phys. Lett. 105, 011123 (2014).

${ }^{25} \mathrm{G}$. Calusine, A. Politi, and D. D. Awschalom, Phys. Rev. Appl. 6, 014019 (2016).

${ }^{26}$ P. J. Dean, J. R. Haynes, and W. F. Flood, Phys. Rev. 161, 711 (1967).

${ }^{27}$ A. Lohrmann, B. C. Johnson, J. C. McCallum, and S. Castelletto, Rep. Prog. Phys. 80, 034502 (2017).

${ }^{28}$ J. Baur, M. Kunzer, and J. Schneider, Phys. Status Solidi A 162, 153 (1997).

${ }^{29}$ K. Sakoda, Optical Properties of Photonic Crystals, Springer Series in Optical Sciences (Springer-Verlag, Berlin/Heidelberg, 2005), Vol. 80.

${ }^{30}$ D. Dussault and P. Hoess, in Optical Science and Technology, The SPIE 49th Annual Meeting, edited by E. L. Dereniak, R. E. Sampson, and C. B. Johnson (SPIE, 2004), p. 195.

${ }^{31}$ S. Ferrari, C. Schuck, and W. Pernice, Nanophotonics 7, 1725 (2018).

${ }^{32}$ J. Münzberg, A. Vetter, F. Beutel, W. Hartmann, S. Ferrari, W. H. P. Pernice, and C. Rockstuhl, Optica 5, 658 (2018).

${ }^{33}$ A. Faraon, I. Fushman, D. Englund, N. Stoltz, P. Petroff, and J. Vuckovic, Nat. Phys. 4, 859 (2008).

${ }^{34}$ M. Galli, S. L. Portalupi, M. Belotti, L. C. Andreani, L. O’Faolain, and T. F. Krauss, Appl. Phys. Lett. 94, 071101 (2009).
${ }^{35}$ K. Demarest, Z. Huang, and R. Plumb, IEEE Trans. Antennas Propag. 44, 1150 (1996).

${ }^{36}$ A. Taflove and S. C. Hagness, Computational Electrodynamics: The FiniteDifference Time-Domain Method, 3rd ed. (Artech House, 2004).

${ }^{37}$ A. R. A. Chalcraft, S. Lam, D. O’Brien, T. F. Krauss, M. Sahin, D. Szymanski, D. Sanvitto, R. Oulton, M. S. Skolnick, A. M. Fox, D. M. Whittaker, H. Y. Liu, and M. Hopkinson, Appl. Phys. Lett. 90, 241117 (2007).

${ }^{38}$ Y. Akahane, T. Asano, B.-S. Song, and S. Noda, Opt. Express 13, 1202 (2005).

${ }^{39}$ N.-V.-Q. Tran, S. Combrié, and A. De Rossi, Phys. Rev. B 79, 041101 (2009).

${ }^{40}$ S. L. Portalupi, M. Galli, C. Reardon, and T. Krauss, Opt. Express 18, 16064 (2010).

${ }^{41}$ A. D. Bailey III, M. C. M. van de Sanden, J. A. Gregus, and R. A. Gottscho, J. Vac. Sci. Technol., B 13, 92 (1995).

${ }^{42}$ L. Novotny and B. Hecht, Principles of Nano-Optics (Cambridge University Press, Cambridge, 2006).

${ }^{43}$ A. M. Kowalevicz, Jr. and F. Bucholtz, "Beam divergence from an SMF-28 optical fiber," Technical Report, 2006.

${ }^{44}$ K. Maeno, Y. Takahashi, T. Nakamura, T. Asano, and S. Noda, Opt. Express 25, 367 (2017).

${ }^{45}$ J. K. Komma, "Optische Eigenschaften von Substratmaterialien für zukünftige kryogene Gravitationswellendetektoren," Ph.D. thesis (Friedrich-SchillerUniversität Jena, 2016).

${ }^{46}$ M. P. Ross, "Bound exciton-assisted spin-to-charge conversion of donors in silicon," Ph.D. thesis (University College London, 2017).

${ }^{47}$ P. J. Dean, W. F. Flood, and G. Kaminsky, Phys. Rev. 163, 721 (1967).

${ }^{48}$ E. M. Purcell, Phys. Rev. 69, 674 (1946), available at http://prola.aps.org/pdf/ PR/v69/i11-12/p674_2.

${ }^{49} \mathrm{We}$ note that the reported Q values of SiC PCCs are low for wavelengths around $1100 \mathrm{~nm}$, attributed to optical losses from fabrication imperfections and absorption from defects and damage in $\mathrm{SiC}$ material. ${ }^{50}$ Our results suggest the approach of coupling SiC defect spins to high Q Si PCCs through selective epitaxial growth (SEG) of SiC thin films ${ }^{51,52}$ on silicon substrates.

${ }^{50}$ B.-S. Song, S. Jeon, H. Kim, D. D. Kang, T. Asano, and S. Noda, Appl. Phys. Lett. 113, 231106 (2018).

${ }^{51}$ K. Teker, J. Cryst. Growth 257, 245 (2003).

${ }^{52}$ A. Gupta and C. Jacob, Prog. Cryst. Growth Charact. Mater. 51, 43 (2005). 